\title{
A Collocation Method for Solving Fractional Riccati Differential Equation
}

\author{
Yalçın Öztürk, ${ }^{1}$ Ayşe Anapalı, ${ }^{2}$ Mustafa Gülsu, ${ }^{2}$ and Mehmet Sezer ${ }^{3}$ \\ ${ }^{1}$ Ula Ali Koçman Vocational Scholl, Muğla Sıtkı Koçman University, 48000 Muğla, Turkey \\ ${ }^{2}$ Department of Mathematics, Faculty of Science, Muğla Sitkı Koçman University, 48000 Muğla, Turkey \\ ${ }^{3}$ Department of Mathematics, Faculty of Science and Arts, Celal Bayar University, 45047 Manisa, Turkey
}

Correspondence should be addressed to Mehmet Sezer; mehmet.sezer@cbu.edu.tr

Received 29 March 2013; Accepted 9 July 2013

Academic Editor: Jafar Biazar

Copyright (C) 2013 Yalçın Öztürk et al. This is an open access article distributed under the Creative Commons Attribution License, which permits unrestricted use, distribution, and reproduction in any medium, provided the original work is properly cited.

\begin{abstract}
We have introduced a Taylor collocation method, which is based on collocation method for solving fractional Riccati differential equation with delay term. This method is based on first taking the truncated Taylor expansions of the solution function in the fractional Riccati differential equation and then substituting their matrix forms into the equation. Using collocation points, we have the system of nonlinear algebraic equation. Then, we solve the system of nonlinear algebraic equation using Maple 13, and we have the coefficients of the truncated Taylor sum. In addition, illustrative examples are presented to demonstrate the effectiveness of the proposed method. Comparing the methodology with some known techniques shows that the present approach is relatively easy and highly accurate.
\end{abstract}

\section{Introduction}

The concept of fractional or noninteger order derivation and integration can be traced back to the genesis of integer order calculus itself $[1,2]$. The recent investigations in science and engineering have demonstrated that the dynamics of many systems may be described more accurately by using differential equations of noninteger order. The fractional differential equations FDEs have shown to be adequate models for various physical phenomena in areas like damping laws, diffusion processes, and so forth. For example, the nonlinear oscillation of earthquake can be modeled with fractional derivatives [3] and the fluid-dynamic traffic model with fractional derivatives [4], psychology [5] and so forth, [6-9].

In this paper, we present numerical and analytical solutions for the fractional Riccati differential equation with delay term

$$
\begin{array}{r}
D_{*}^{\alpha} y(x)=A(x)+B(x) y(\lambda x+\beta)+C(x) y^{2}(x), \\
x>0,0<\alpha \leq 1
\end{array}
$$

subject to the initial conditions

$$
y(0)=\lambda,
$$

where $A(x), B(x)$, and $C(x)$ are given functions, $\alpha$ is a parameter describing the order of the fractional derivative and $\lambda, \beta$ are appropriate constants, and $\lambda x+\beta>0$ for all $x \in$ $[0,1]$. The general response expression contains a parameter describing the order of the fractional derivative that can be varied to obtain various responses. In the case of $\alpha=1$, the fractional equation reduces to the classical Riccati differential equation. The importance of this equation usually arises in the optimal control problems [10]. The existing literature on fractional differential equations tends to focus on particular values for the order $\alpha$. In modern applications (see e.g., [11]) much more general values of the order appear in the equations, and therefore one needs to consider numerical and analytical methods to solve differential equations of arbitrary order. This equation is solved the numerically in [12-14]. 
We seek the approximate solution of (1) under the conditions (2) with the fractional Taylor series as $D_{a}^{k \alpha} y(x) \in$ $C(a, b]$,

$$
y_{N}(x)=\sum_{i=0}^{N} \frac{(x-a)^{i \alpha}}{\Gamma(i \alpha+1)}\left(D_{a}^{i \alpha} y(x)\right)_{x=a}
$$

where $0<\alpha \leq 1$. In recently, collocation method has become a very useful technique for solving equations [15-22]. This method transforms each part of the equation into matrix form and using the collocation points as

$$
x_{i}=\frac{i}{N}, \quad i=0,1, \ldots, N,
$$

we get the nonlinear algebraic equation. Then this equation is solved, we obtained the coefficients, the approximate solutions for various $N$. All computations are performed on the computer algebraic system Maple 13 in this paper.

\section{Basic Definitions}

In this section, we first give some basic definitions and then present properties of fractional calculus $[1,2,23]$.

Definition 1. A real function $f(x), x>0$ is said to be in space $C_{\mu}, \mu \in R \in$ if there exists a real number $p(>\mu)$, such that $f(x)=x^{p} f_{1}(x)$, where $f_{1}(x) \in[0, \infty)$, and it is said to be in the space $C_{\mu}^{m}$ iff $f^{(m)} \in C_{\mu}, m \in N$.

Definition 2. The Riemann-Liouville fractional derivative of order $\alpha$ with respect to the variable $t$ and with the starting point at $x=a$ is

$$
\begin{aligned}
& { }_{a} D_{x}^{\alpha} f(x) \\
& = \begin{cases}\frac{1}{\Gamma(-\alpha+m+1)} \frac{d^{m+1}}{d x^{m+1}} & \\
\times \int_{a}^{x}(x-\tau)^{m-\alpha} f(\tau) d \tau, & 0 \leq m \leq \alpha<m+1 \\
\frac{d^{m} f(x)}{d t^{m}}, & \alpha=m+1 \in N .\end{cases}
\end{aligned}
$$

Definition 3. The Riemann-Liouville fractional integral of order $\alpha$ is

$$
{ }_{a} D_{x}^{-\alpha} f(x)=\frac{1}{\Gamma(\alpha)} \int_{a}^{x}(x-\tau)^{\alpha-1} f(\tau) d \tau, \quad p>0 .
$$

Definition 4. The fractional derivative of $f(x)$ by means of Caputo sense is defined as

$$
D^{\alpha} f(x)=\frac{1}{\Gamma(n-\alpha)} \int_{0}^{x}(x-\tau)^{n-\alpha-1} f^{(n)}(\tau) d \tau
$$

for $n-1<\alpha \leq n, n \in N, x>0, f \in C_{-1}^{n}$.
For the Caputo's derivative we have $D^{\alpha} C=0, C$ is a constant,

$$
D^{\alpha} x^{n}= \begin{cases}0, & n \in N, n<\lceil\alpha\rceil \\ \frac{\Gamma(n+1)}{\Gamma(n+1-\alpha)} x^{n-\alpha}, & n \in N, n<\lfloor\alpha\rfloor .\end{cases}
$$

Theorem 5 (generalized Taylor formula). Suppose that $D_{a}^{k \alpha} f(x) \in C(a, b]$ for $k=0,1, \ldots, n+1$, where $0<\alpha \leq 1$, then one has [23]

$$
\begin{aligned}
f(x)= & \sum_{i=0}^{n} \frac{(x-a)^{i \alpha}}{\Gamma(i \alpha+1)}\left(D_{a}^{i \alpha} f\right)(a) \\
& +\frac{\left(D_{a}^{(n+1)} f\right)(\xi)}{\Gamma((n+1) \alpha+1)}(x-a)^{(n+1) \alpha}
\end{aligned}
$$

with $a \leq \xi \leq x$, for all $x \in(a, b]$, where

$$
D_{a}^{n \alpha}=D_{a}^{\alpha} \cdot D_{a}^{\alpha} \cdot D_{a}^{\alpha} \cdots D_{a}^{\alpha} \quad(n \text { times }) .
$$

\section{Fundamental Relations}

In this section, we consider the fractional Ricatti differential equations (1). We use the Taylor matrix method [15-22] to find the truncated Taylor series expansions of each term in expression at $x=c$ and their matrix representations for solving $\alpha$ th order linear fractional part and nonlinear part. We first consider the solution $y(x)$ of (1) defined by a truncated Taylor series (3). Then, we have the matrix form of the solution $y_{N}(x)$

$$
\left[y_{N}(x)\right]=\mathbf{T}(x) \mathbf{A}=\mathbf{X}(x) \mathbf{M}_{0} \mathbf{A},
$$

where

$$
\begin{gathered}
\mathbf{X}(x)=\left[\begin{array}{lllll}
1 & (x-c)^{\alpha} & (x-c)^{2 \alpha} & \cdots & (x-c)^{N \alpha}
\end{array}\right], \\
\mathbf{M}_{0}=\left[\begin{array}{ccccc}
\frac{1}{\Gamma(1)} & 0 & 0 & \cdots & 0 \\
0 & \frac{1}{\Gamma(\alpha+1)} & 0 & \cdots & 0 \\
0 & 0 & \frac{1}{\Gamma(2 \alpha+1)} & \cdots & 0 \\
\vdots & \vdots & \vdots & \ddots & \vdots \\
0 & 0 & 0 & \cdots & \frac{1}{\Gamma(N \alpha+1)}
\end{array}\right], \\
\mathbf{A}=\left[\begin{array}{c}
D_{*}^{0 \alpha} y(c) \\
D_{*}^{1 \alpha} y(c) \\
D_{*}^{2 \alpha} y(c) \\
\vdots \\
D_{*}^{N \alpha} y(c)
\end{array}\right] .
\end{gathered}
$$


Then, the matrix representation of the function $D_{*}^{\alpha} y_{N}(x) \quad$ where we compute the $D_{*}^{\alpha} \mathbf{X}(x)$, then becomes

$$
D_{*}^{\alpha} y_{N}(x)=D_{*}^{\alpha} \mathbf{X}(x) \mathbf{M}_{0} \mathbf{A}
$$

$$
\begin{aligned}
& D_{*}^{\alpha} \mathbf{X}(x)=\left[\begin{array}{lllll}
D_{*}^{\alpha} 1 & D_{*}^{\alpha}(x-c)^{\alpha} & D_{*}^{\alpha}(x-c)^{2 \alpha} & \cdots & D_{*}^{\alpha}(x-c)^{N \alpha}
\end{array}\right] \\
& =\left[\begin{array}{llll}
0 & \frac{\Gamma(\alpha+1)}{\Gamma(1)} & \frac{\Gamma(2 \alpha+1)}{\Gamma(\alpha+1)}(x-c)^{\alpha} & \cdots
\end{array} \frac{\Gamma(N \alpha+1)}{\Gamma((N-1) \alpha+1)}(x-c)^{(N-1) \alpha}\right] \\
& =\mathbf{X}(x) \mathbf{M}_{1} \text {, }
\end{aligned}
$$

where

$$
\mathbf{M}_{1}=\left[\begin{array}{ccccc}
0 & \frac{\Gamma(\alpha+1)}{\Gamma(1)} & 0 & \cdots & 0 \\
0 & 0 & \frac{\Gamma(2 \alpha+1)}{\Gamma(\alpha+1)} & \cdots & 0 \\
\vdots & \vdots & \vdots & \ddots & \vdots \\
0 & 0 & 0 & \cdots & \frac{\Gamma(N \alpha+1)}{\Gamma((N-1) \alpha+1)} \\
0 & 0 & 0 & \cdots & 0
\end{array}\right] .
$$

Then, so the matrix representation of fractional differential part as

$$
D_{*}^{\alpha} y_{N}(x)=\mathbf{X}(x) \mathbf{M}_{1} \mathbf{M}_{0} \mathbf{A}
$$

Additionally, using (11) we can write

$$
\left[y_{N}(\lambda x+\beta)\right]=\mathbf{X}(\lambda x+\beta) \mathbf{M}_{0} \mathbf{A}=\overline{\mathbf{X}}(x) \mathbf{M}_{0} \mathbf{A},
$$

where

$$
\begin{aligned}
& \overline{\mathbf{X}}(x)=\mathbf{X}(\lambda x+\beta) \\
& =\left[\begin{array}{llll}
1 & (\lambda x+\beta-c)^{\alpha} & \cdots & (\lambda x+\beta-c)^{N \alpha}
\end{array}\right] .
\end{aligned}
$$

Moreover, since [21, 22]

$$
\mathbf{Y}^{m}=\mathbf{Y}^{m-1} \overline{\mathbf{Y}}
$$

where

$$
\begin{gathered}
\mathbf{Y}^{m-1}(x)=\left[\begin{array}{c}
y^{m-1}(x) \\
y^{m-1}(x) \\
\vdots \\
y^{m-1}(x)
\end{array}\right], \\
\overline{\mathbf{Y}}(x)=\left[\begin{array}{cccc}
y(x) & 0 & \cdots & 0 \\
0 & y(x) & \cdots & 0 \\
\vdots & \vdots & \ddots & \vdots \\
0 & 0 & \cdots & y(x)
\end{array}\right],
\end{gathered}
$$

and using collocation points in (11)

$$
\overline{\mathbf{Y}}=\overline{\mathbf{T}} \overline{\mathbf{A}},
$$

where

$$
\overline{\mathbf{T}}(x)=\left[\begin{array}{cccc}
\mathbf{T}(x) & 0 & \cdots & 0 \\
0 & \mathbf{T}(x) & \cdots & 0 \\
\vdots & \vdots & \ddots & \vdots \\
0 & 0 & \cdots & \mathbf{T}(x)
\end{array}\right],
$$

$$
\overline{\mathbf{A}}=\left[\begin{array}{cccc}
\mathbf{A} & 0 & \cdots & 0 \\
0 & \mathbf{A} & \cdots & 0 \\
\vdots & \vdots & \ddots & \vdots \\
0 & 0 & \cdots & \mathbf{A}
\end{array}\right]
$$

then we construct the following relation

$$
y\left(x_{i}\right) y\left(x_{i}\right)=y\left(x_{i}\right) y\left(x_{i}\right)=(\overline{\mathbf{T}} \overline{\mathbf{A}}) \mathbf{X}\left(x_{i}\right) \mathbf{M}_{0} \mathbf{A} .
$$

Hence, the fundamental matrix relation of (1) is

$$
\begin{aligned}
& \left(\mathbf{X}(x) \mathbf{M}_{1} \mathbf{M}_{0}-B(x) \overline{\mathbf{X}}(x) \mathbf{M}_{0}\right. \\
& \left.\quad-C(x)(\overline{\mathbf{T}} \overline{\mathbf{A}}) \mathbf{X}(x) \mathbf{M}_{0}\right) \mathbf{A}=A(x) .
\end{aligned}
$$

Finally, we obtained matrix representation of the condition in (2)

$$
\mathbf{U}_{0}=\mathbf{X}(0) \mathbf{M}_{0} \mathbf{A}=\left[\begin{array}{lllll}
u_{0} & u_{1} & u_{2} & \cdots & u_{N}
\end{array}\right]=[\lambda] .
$$

\section{Method of Solution}

Using collocation points in (4), we can write (24)

$$
\begin{aligned}
& \left(\mathbf{X}\left(x_{i}\right) \mathbf{M}_{1} \mathbf{M}_{0}-B\left(x_{i}\right) \overline{\mathbf{X}}\left(x_{i}\right) \mathbf{M}_{0}\right. \\
& \left.\quad-C\left(x_{i}\right)(\overline{\mathbf{T}} \overline{\mathbf{A}}) \mathbf{X}\left(x_{i}\right) \mathbf{M}_{0}\right) \mathbf{A}=A\left(x_{i}\right)
\end{aligned}
$$

or briefly the fundamental matrix equation

$$
\left(\mathbf{X M}_{1} \mathbf{M}_{0}-\mathbf{B} \overline{\mathbf{X}} \mathbf{M}_{0}-\mathbf{C}(\overline{\mathbf{T}} \overline{\mathbf{A}}) \mathbf{X} \mathbf{M}_{0}\right) \mathbf{A}=\mathbf{F},
$$

where 


$$
\begin{aligned}
& \mathbf{X}=\left[\begin{array}{ccccc}
1 & \left(x_{0}-c\right)^{\alpha} & \left(x_{0}-c\right)^{2 \alpha} & \ldots & \left(x_{0}-c\right)^{N \alpha} \\
1 & \left(x_{1}-c\right)^{\alpha} & \left(x_{1}-c\right)^{2 \alpha} & \ldots & \left(x_{1}-c\right)^{N \alpha} \\
1 & \left(x_{2}-c\right)^{\alpha} & \left(x_{2}-c\right)^{2 \alpha} & \ldots & \left(x_{2}-c\right)^{N \alpha} \\
\vdots & \vdots & \vdots & \ddots & \vdots \\
1 & \left(x_{N}-c\right)^{\alpha} & \left(x_{N}-c\right)^{2 \alpha} & \cdots & \left(x_{N}-c\right)^{N \alpha}
\end{array}\right], \quad \mathbf{F}=\left[\begin{array}{c}
A\left(x_{0}\right) \\
A\left(x_{1}\right) \\
A\left(x_{2}\right) \\
\vdots \\
A\left(x_{N}\right)
\end{array}\right] \\
& \overline{\mathbf{X}}=\left[\begin{array}{ccccc}
1 & \left(\lambda x_{0}+\beta-c\right)^{\alpha} & \left(\lambda x_{0}+\beta-c\right)^{2 \alpha} & \ldots & \left(\lambda x_{0}+\beta-c\right)^{N \alpha} \\
1 & \left(\lambda x_{1}+\beta-c\right)^{\alpha} & \left(\lambda x_{1}+\beta-c\right)^{2 \alpha} & \ldots & \left(\lambda x_{1}+\beta-c\right)^{N \alpha} \\
1 & \left(\lambda x_{2}+\beta-c\right)^{\alpha} & \left(\lambda x_{2}+\beta-c\right)^{2 \alpha} & \ldots & \left(\lambda x_{2}+\beta-c\right)^{N \alpha} \\
\vdots & \vdots & \vdots & \ddots & \vdots \\
1 & \left(\lambda x_{N}+\beta-c\right)^{\alpha} & \left(\lambda x_{N}+\beta-c\right)^{2 \alpha} & \ldots & \left(\lambda x_{N}+\beta-c\right)^{N \alpha}
\end{array}\right] \\
& \overline{\mathbf{T}}=\left[\begin{array}{ccccc}
T\left(x_{0}\right) & 0 & 0 & \cdots & 0 \\
0 & T\left(x_{1}\right) & 0 & \cdots & 0 \\
0 & 0 & T\left(x_{2}\right) & \cdots & 0 \\
\vdots & \vdots & \vdots & \ddots & \vdots \\
0 & 0 & 0 & \cdots & T\left(x_{N}\right)
\end{array}\right] \\
& \mathbf{B}=\left[\begin{array}{ccccc}
B\left(x_{0}\right) & 0 & 0 & \cdots & 0 \\
0 & B\left(x_{1}\right) & 0 & \cdots & 0 \\
0 & 0 & B\left(x_{2}\right) & \cdots & 0 \\
\vdots & \vdots & \vdots & \ddots & \vdots \\
0 & 0 & 0 & \cdots & B\left(x_{N}\right)
\end{array}\right] \\
& \mathbf{C}=\left[\begin{array}{ccccc}
C\left(x_{0}\right) & 0 & 0 & \cdots & 0 \\
0 & C\left(x_{1}\right) & 0 & \cdots & 0 \\
0 & 0 & C\left(x_{2}\right) & \cdots & 0 \\
\vdots & \vdots & \vdots & \ddots & \vdots \\
0 & 0 & 0 & \cdots & C\left(x_{N}\right)
\end{array}\right]
\end{aligned}
$$

Hence, the fundamental matrix equation (27) corresponding to (1) can be written in the form

$$
\mathbf{W A}=\mathbf{F} \quad \text { or } \quad[\mathbf{W} ; \mathbf{F}], \mathbf{W}=\left[w_{i, j}\right], i, j=0,1, \ldots, N \text {, }
$$

where

$$
\mathbf{W}=\mathbf{X} \mathbf{M}_{1} \mathbf{M}_{0}-\mathbf{B} \overline{\mathbf{X}} \mathbf{M}_{0}-\mathbf{C}(\overline{\mathbf{T}} \overline{\mathbf{A}}) \mathbf{X} \mathbf{M}_{0} .
$$

To obtain the solution of (1) under conditions (2), by replacing the row matrices (25) by the last 2 rows of the matrix (26), we have the new augmented matrix,

$$
[\widetilde{\mathbf{W}} ; \widetilde{\mathbf{F}}]=\left[\begin{array}{cccccc}
w_{00} & w_{01} & \cdots & w_{0 N} & ; & A\left(x_{0}\right) \\
w_{10} & w_{11} & \cdots & w_{1 N} & ; & A\left(x_{1}\right) \\
\vdots & \vdots & \ddots & \vdots & \vdots & \vdots \\
& & & & \vdots & A\left(x_{N-2}\right) \\
w_{N-20} & w_{N-21} & \cdots & w_{N-2 N} & ; & A\left(x_{N-1}\right) \\
w_{N-10} & w_{N-11} & \cdots & w_{N-1 N} & ; & \lambda \\
u_{0} & u_{1} & \cdots & u_{N} & ; & \lambda
\end{array}\right]
$$

So, we obtained a system of $(N+1)$ nonlinear algebraic equations with unknown Taylor coefficients.

We can easily check the accuracy of the method. Since the truncated fractional Taylor series (3) is an approximate solution of (1), when the solution $y_{N}(x)$ and its derivatives are substituted in (1), the resulting equation must be satisfied approximately; that is, for $x=x_{q} \in[0,1], q=0,1,2, \ldots$

$$
\begin{gathered}
E_{N}\left(x_{q}\right)=\mid D_{*}^{\alpha} y\left(x_{q}\right)-A\left(x_{q}\right)-B\left(x_{q}\right) y\left(\lambda x_{q}+\beta\right) \\
-C\left(x_{q}\right) y^{2}\left(x_{q}\right) \mid \cong 0 .
\end{gathered}
$$

\section{Examples}

In order to illustrate the effectiveness of the method proposed in this paper, several numerical examples are carried out in this section. In the following computations, for convenience, absolute errors between $N$ th-order approximate values $y_{N}$ and the corresponding exact values $y_{\mathrm{ex}}$ as $N_{e}=\left|y_{N}-y_{\mathrm{ex}}\right|$ are determined and all computations performed computer algebraic system with mathematical programing in Maple 13.

Example 1. Consider the following fractional Riccati equation:

$$
\begin{array}{r}
D_{*}^{\alpha} y(x)=y^{2}(x)-x^{2} y(x+1)+\frac{\Gamma(3)}{\Gamma(5 / 2)} x^{3 / 2}-2 x^{3}-x^{2}, \\
x>0,0<\alpha \leq 1
\end{array}
$$

with initial conditions

$$
y(0)=0
$$




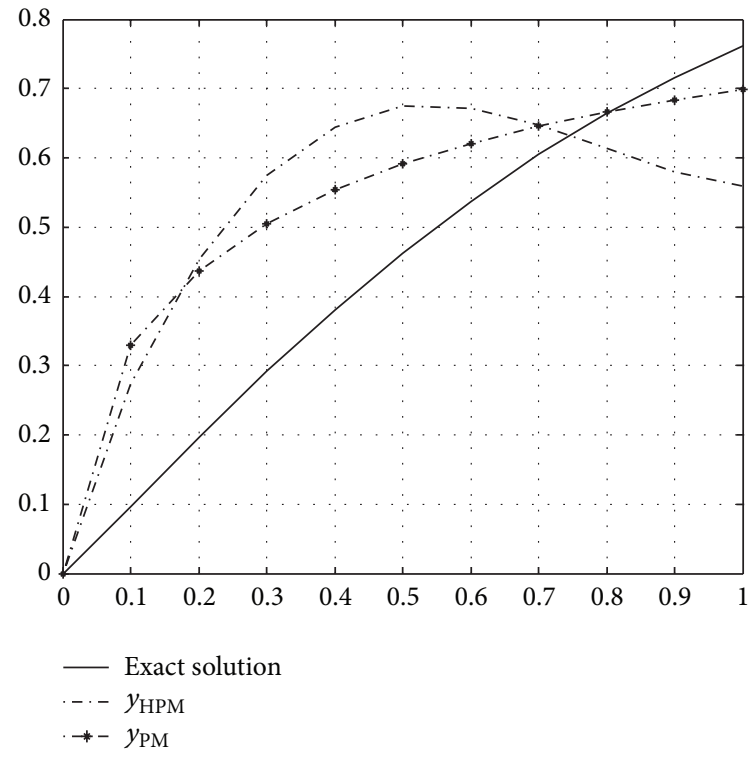

FIgURE 1: Comparison of the HPM and PMN for $\alpha=0.5$.

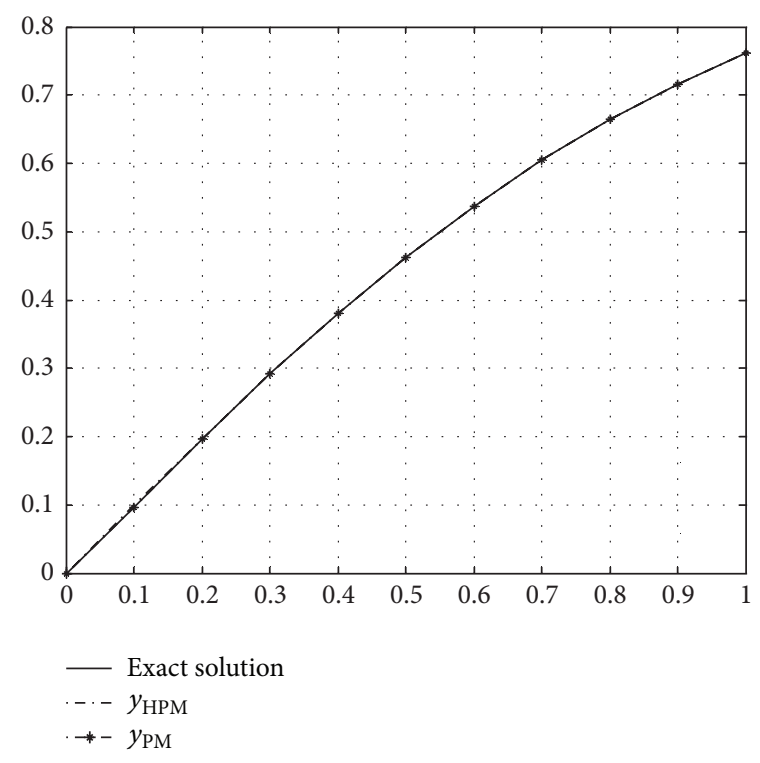

FIGURE 2: Comparison of the HPM and PM for $\alpha=1$.

Then, $A(x)=\Gamma(3) x^{3 / 2} / \Gamma(5 / 2)-2 x^{3}-x^{2}, B(x)=-x^{2}, C(x)=$ 1. We assume that $\alpha=1 / 2,0 \leq x \leq 1$, and we seek the approximate solutions $y_{N}$ by Taylor series, for $c=0, N=4$

$$
y_{4}(x)=\sum_{k=0}^{4} \frac{x^{k \alpha}}{\Gamma(k \alpha+1)}\left(D_{*}^{k \alpha} y(x)\right)_{x=0}
$$

with collocation points being

$$
\begin{gathered}
x_{0}=0, \quad x_{1}=\frac{1}{4}, \\
x_{2}=\frac{2}{4}, \quad x_{3}=\frac{3}{4}, \\
x_{4}=1 .
\end{gathered}
$$

Fundamental matrix relation of this problem is

$$
\left(\mathbf{X M}_{1} \mathbf{M}_{0}-\mathbf{B} \overline{\mathbf{X}} \mathbf{M}_{0}-\mathbf{C}(\overline{\mathbf{T}} \overline{\mathbf{A}}) \mathbf{X} \mathbf{M}_{0}\right) \mathbf{A}=\mathbf{F}
$$

where

$$
\mathbf{X}=\left[\begin{array}{ccccc}
1 & 0 & 0 & 0 & 0 \\
1 & \frac{1}{\sqrt{4}} & \frac{1}{4} & \frac{\sqrt{4}}{16} & \frac{1}{16} \\
1 & \frac{1}{\sqrt{2}} & \frac{1}{2} & \frac{\sqrt{2}}{4} & \frac{1}{4} \\
1 & \frac{\sqrt{3}}{\sqrt{4}} & \frac{3}{4} & \frac{3 \sqrt{3}}{4 \sqrt{4}} & \frac{9}{16} \\
1 & 1 & 1 & 1 & 1
\end{array}\right], \quad \mathbf{B}=\left[\begin{array}{ccccc}
1 & 0 & 0 & 0 & 0 \\
0 & 1 & 0 & 0 & 0 \\
0 & 0 & 1 & 0 & 0 \\
0 & 0 & 0 & 1 & 0 \\
0 & 0 & 0 & 0 & 1
\end{array}\right]
$$

$$
\overline{\mathbf{X}}=\left[\begin{array}{cccccc}
1 & 2 & 2 & 2 & 2 \\
1 & \frac{1}{\sqrt{4}}+1 & \frac{1}{4}+1 & \frac{\sqrt{4}}{16}+1 & \frac{1}{16}+1 \\
1 & \frac{1}{\sqrt{2}}+1 & \frac{1}{2}+1 & \frac{\sqrt{2}}{4}+1 & \frac{1}{4}+1 \\
1 & \frac{\sqrt{3}}{\sqrt{4}}+1 & \frac{3}{4}+1 & \frac{3 \sqrt{3}}{4 \sqrt{4}}+1 & \frac{9}{16}+1 \\
1 & 2 & 2 & 2 & 2
\end{array}\right],
$$$$
\mathbf{M}_{1}=\left[\begin{array}{ccccc}
0 & 1 & 0 & 0 & 0 \\
0 & 0 & \frac{2}{\sqrt{\pi}} & 0 & 0 \\
0 & 0 & 0 & 1 & 0 \\
0 & 0 & 0 & 0 & \frac{4}{3 \sqrt{\pi}} \\
0 & 0 & 0 & 0 & 0
\end{array}\right], \quad \mathbf{F}=\left[\begin{array}{c}
0.000000 \\
0.188063 \\
0.531923 \\
0.977205 \\
1.504505
\end{array}\right]
$$

Also, we have the matrix representation of conditions,

$$
y(0)=\left[\begin{array}{lllllll}
1 & 0 & 0 & 0 & 0 & 0 & 0
\end{array}\right] \mathbf{A}=[0]
$$


TABLE 1: Numerical result for Example 1.

\begin{tabular}{cccccccc}
\hline$x$ & Exact solution & $N=4$ & $N_{e}=4$ & $N=5$ & $N_{e}=5$ & $N=6$ & $N_{e}=6$ \\
\hline 0.0 & 0.000 & 0.000000 & $0.000 E-0$ & 0.000000 & $0.000 E-0$ & 0.00000 \\
0.2 & 0.040 & 0.039985 & $0.150 E-4$ & 0.040000 & $0.553 E-6$ & 0.040000 & $0.000 E-0$ \\
0.4 & 0.160 & 0.159993 & $0.700 E-5$ & 0.160000 & $0.352 E-6$ & 0.160000 & $0.530 E-8$ \\
0.6 & 0.360 & 0.359996 & $0.446 E-5$ & 0.360000 & $0.330 E-6$ & 0.360000 & $0.497 E-8$ \\
0.8 & 0.640 & 0.63996 & $0.446 E-5$ & 0.640000 & $0.337 E-6$ & 0.640000 & $0.528 E-8$ \\
1.0 & 1.000 & 0.999998 & $0.219 E-5$ & 1.000000 & $0.464 E-6$ & 1.000000 & $0.673 E-8$ \\
\hline
\end{tabular}

TABLE 2: The numerical results with comparison HP and PM $(N=12)$.

\begin{tabular}{|c|c|c|c|c|c|}
\hline \multirow{2}{*}{$x$} & \multirow{2}{*}{$y_{\text {exact }}$} & \multicolumn{2}{|c|}{$y_{\mathrm{HPM}}$} & \multicolumn{2}{|c|}{$y_{\mathrm{PM}}$} \\
\hline & & $\alpha=0.5$ & $\alpha=1$ & $\alpha=0.5$ & $\alpha=1$ \\
\hline 0.0 & 0.000000 & 0.000000 & 0.000000 & 0.000000 & 0.000000 \\
\hline 0.1 & 0.096668 & 0.273875 & 0.099668 & 0.329929 & 0.096668 \\
\hline 0.2 & 0.197375 & 0.454125 & 0.197375 & 0.436741 & 0.197375 \\
\hline 0.3 & 0.291313 & 0.573932 & 0.291312 & 0.504823 & 0.291313 \\
\hline 0.4 & 0.379949 & 0.644422 & 0.379944 & 0.553733 & 0.379949 \\
\hline 0.5 & 0.462117 & 0.674137 & 0.462078 & 0.591156 & 0.462117 \\
\hline 0.6 & 0.537050 & 0.671987 & 0.536857 & 0.620983 & 0.537050 \\
\hline 0.7 & 0.604368 & 0.648003 & 0.603631 & 0.645461 & 0.604368 \\
\hline 0.8 & 0.664037 & 0.613306 & 0.661706 & 0.665998 & 0.664037 \\
\hline 0.9 & 0.716298 & 0.579641 & 0.709919 & 0.683545 & 0.716298 \\
\hline 1.0 & 0.761594 & 0.558557 & 0.746032 & 0.698852 & 0.761594 \\
\hline
\end{tabular}

and so we solve (37) and obtain the coefficients of the Taylor series

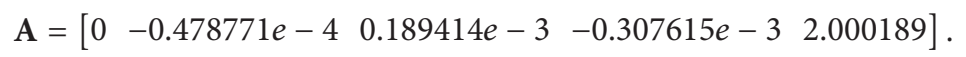

Hence, for $N=4$, the approximate solution of Example 1 is given as

$$
\begin{aligned}
y_{5}= & \frac{0.957543 \sqrt{x}}{\sqrt{\pi}}+0.189414 e-3 x \\
& -\frac{0.410153 x^{3 / 2}}{\sqrt{\pi}}+1.000094 x^{2} .
\end{aligned}
$$

Comparison of numerical results with the exact solution is shown in Table 1 for various $N$.

Example 2. Let us consider the following fractional Riccati equation [13]

$$
D_{*}^{\alpha} y(x)=y^{2}(x)+1, \quad 0<\alpha \leq 1
$$

subject to the initial condition

$$
y(0)=1 \text {. }
$$

Then, $A(x)=1, B(x)=0, C(x)=1$. Fundamental matrix relation of this problem is

$$
\left(\mathbf{X M}_{1} \mathbf{M}_{0}-\mathbf{C}(\overline{\mathbf{T}} \overline{\mathbf{A}}) \mathbf{X M}_{0}\right) \mathbf{A}=\mathbf{F} .
$$

Also, we have the matrix representation of conditions,

$$
y(0)=\left[\begin{array}{lllllll}
1 & 0 & 0 & 0 & 0 & 0 & 0
\end{array}\right] \mathbf{A}=[0] .
$$

The exact solution, when $\alpha=1$, is 
We approximately solve the fractional Ricatti equation for $N=12$ and obtained the approximate solution for $\alpha=1$,

$$
\begin{aligned}
y_{12}= & x-0.559171 e-6 x^{2}-0.333319 x^{3}-0.155476 e \\
& -3 x^{4}+0.134388 x^{5}-0.467422 e-2 x^{6}-0.398472 e \\
& -1 x^{7}-0.295050 e-1 x^{8}+0.640148 e \\
& -1 x^{9}-0.390402 e-1 x^{10}+0.109027 x^{11} \\
& -0.116904 e-2 x^{12} .
\end{aligned}
$$

Table 2 shows the approximate solutions for Example 2 obtained by the present method and compare with homotopy perturbation method for different $\alpha$. From the numerical results in Table 2, present method is in high agreement with the exact solution than homotopy perturbation method [13]. Moreover, using the numerical result in Table 2, Figures 1 and 2 are plotted. Figure 2 shows that numerical solution $y_{\mathrm{PM}}$ is so closed to the exact solution.

\section{Conclusion}

In real world systems, delays can be recognised everywhere and there has been widespread interest in the study of delay differential equations for many years. Although it seems natural to model certain processes and systems in engineering and other sciences with this kind of equation, only in the last few years has the attention of the scientific community been devoted to them.

In this study, we present a Taylor collocation method for the numerical solutions of fractional Riccati differential equation with delay term. This method transforms fractional Riccati differential equation with delay term into matrix equations. The desired approximate solutions can be determined by solving the resulting system, which can be effectively computed using symbolic computing codes on Maple 13. Examples show that Taylor collocation method has been successfully applied to find the approximate solutions of the fractional Riccati differential equation. Graphics and tables show that this method is extremely effective and practical for this sort of approximate solutions.

\section{References}

[1] J. D. Munkhammar, "Fractional calculus and the Taylor-Riemann series," Undergraduate Mathematics Journal, vol. 6, no. 1, pp. 1-19, 2005.

[2] I. Podlubny, Fractional differential equations, vol. 198 of Mathematics in Science and Engineering, Academic Press, New York, NY, USA, 1999.

[3] J. H. He, "Nonlinear oscillation with fractional derivative and its applications," in International Conference on Vibrating Engineering, Dalian, China, 1998.

[4] J. H. He, "Some applications of nonlinear fractional differential equations and their approximations," Bulletin of Science, Technology \& Society, vol. 15, no. 2, pp. 86-90, 1999.
[5] W. M. Ahmad and R. El-Khazali, "Fractional-order dynamical models of love," Chaos, Solitons and Fractals, vol. 33, no. 4, pp. 1367-1375, 2007.

[6] R. L. Bagley and P. J. Torvik, "On the appearance of the fractional derivative in the behavior of real materials," Journal of Applied Mechanics, vol. 51, no. 2, pp. 294-298, 1984.

[7] K. Diethelm and N. J. Ford, "The numerical solution of linear and nonlinear fractional differential equations involving fractional derivatives of several orders," Numerical Analysis Report 379, Manchester Center for Computational Mathematics, 2001.

[8] J. H. He, "A new approach to nonlinear partial differential equations," Communications in Nonlinear Science and Numerical Simulation, vol. 2, no. 4, pp. 230-235, 1997.

[9] J.-H. He, "Approximate analytical solution for seepage flow with fractional derivatives in porous media," Computer Methods in Applied Mechanics and Engineering, vol. 167, no. 1-2, pp. 57-68, 1998.

[10] B. D. Anderson and J. B. Moore, Optimal Control-Linear Quadratic Methods, Prentice-Hall, New Jersey, NJ, USA, 1990.

[11] R. Gorenflo, "Afterthoughts on interpretation of fractional derivatives and integrals," in Transform Methods and Special Functions, Varna 96, P. Rusev, I. Dimovski, and V. Kiryakova, Eds., Academy of Sciences, Institute of Mathematics and Informatics, Bulgarian, Sofia, 1998.

[12] Y. Keskin, O. Karaoğlu, S. Servi, and G. Oturanç, “The approximate solution of high-order linear fractional differential equations with variable coefficients in terms of generalized Taylor polynomials," Mathematical \& Computational Applications, vol. 16, no. 3, pp. 617-629, 2011.

[13] Z. Odibat and S. Momani, "Modified homotopy perturbation method: application to quadratic Riccati differential equation of fractional order," Chaos, Solitons \& Fractals, vol. 36, no. 1, pp. 167-174, 2008.

[14] S. Momani and N. Shawagfeh, "Decomposition method for solving fractional Riccati differential equations," Applied Mathematics and Computation, vol. 182, no. 2, pp. 1083-1092, 2006.

[15] M. Gülsu and M. Sezer, "A Taylor polynomial approach for solving differential-difference equations," Journal of Computational and Applied Mathematics, vol. 186, no. 2, pp. 349-364, 2006.

[16] M. Sezer, "Taylor polynomial solutions of Volterra integral equations," International Journal of Mathematical Education in Science and Technology, vol. 25, no. 5, pp. 625-633, 1994.

[17] A. Karamete and M. Sezer, "A Taylor collocation method for the solution of linear integro-differential equations," International Journal of Computer Mathematics, vol. 79, no. 9, pp. 987-1000, 2002.

[18] M. Gülsu and M. Sezer, "Approximations to the solution of linear Fredholm integrodifferential-difference equation of high order," Journal of the Franklin Institute, vol. 343, no. 7, pp. 720737, 2006.

[19] M. Sezer and M. Gülsu, "Polynomial solution of the most general linear Fredholm-Volterra integrodifferential-difference equations by means of Taylor collocation method," Applied Mathematics and Computation, vol. 185, no. 1, pp. 646-657, 2007.

[20] M. Gülsu and Y. Öztürk, "A new collocation method for solution of mixed linear integro-differential-difference equations," Applied Mathematics and Computation, vol. 216, no. 7, pp. 21832198, 2010.

[21] M. Gülsu, Y. Öztürk, and M. Sezer, "On the solution of the Abel equation of the second kind by the shifted Chebyshev polynomials," Applied Mathematics and Computation, vol. 217, no. 9, pp. 4827-4833, 2011. 
[22] A. Akyüz-Daşcioğlu and H. Çerdik-Yaslan, "The solution of high-order nonlinear ordinary differential equations by Chebyshev series," Applied Mathematics and Computation, vol. 217, no. 12, pp. 5658-5666, 2011.

[23] Z. M. Odibat and N. T. Shawagfeh, "Generalized Taylor's formula," Applied Mathematics and Computation, vol. 186, no. 1, pp. 286-293, 2007. 